\title{
FNDC5/Irisin: A New Protagonist in Acute Brain Injury
}

\author{
Tianqi Tu, Jianhua Peng, ${ }^{1-3}$ and Yong Jiang ${ }^{1,2,4}$
}

Acute brain injury (ABI), including stroke and traumatic brain injury, which accounts for a heavy burden of morbidity and mortality, results in a great loss of productive life and serious health problem worldwide. To improve patients' survival and ameliorate their outcome, effective clinical treatment strategies and new therapeutic targets are urgently needed. Novel therapeutic strategies, especially about stem cell therapy and various targets to enhance the therapeutic effects of stem cell therapy, are emerging all the time. Irisin is a myokine released from cleavage of the membrane-bound precursor protein fibronectin type III domain-containing protein 5 (FNDC5). This myokine is closely related to metabolism and neural stem cell differentiation, and it gained attention in recent years. Recent studies reported that FNDC5/Irisin may play a key role in brain injury, for it might be a powerful agent mediating the neural cell response and stem cell therapy. This review aims at understanding the newly discovered myokine "Irisin," discussing the potential role and mechanisms of Irisin in $\mathrm{ABI}$, and shedding light on its potential application in stem cell therapy. Furthermore, we propose that Irisin could be a novel protagonist in the treatment and prevention of ABI.

Keywords: acute brain injury, traumatic brain injury, stroke, FNDC5/Irisin, stem cell therapy

\section{Introduction}

$I^{\prime}$ RISIN, CLEAVED FROM FIBRONECTIN type III domaincontaining protein 5 (FNDC5), is identified as a myokine that is released into the circulation after physical exercise [1]. Closely related to energy metabolism, this myokine is capable of stimulating adipocyte browning and thermogenesis in mice and humans [2-4]. As the cleaved and secreted form of FNDC5, Irisin is initially discovered to be secreted by muscle [5]. Then, subsequent studies and experiments confirmed that, in addition to the fact that muscles could secrete Irisin, Irisin could also be found in various brain regions and cellular groups, such as Purkinje cells in cerebellum, astrocytes in hippocampus, neurons in cerebrum, the hypothalamus, and even in cerebrospinal fluid (CSF) [6-10].

Recently, since many significant roles against neurological deficits of Irisin peptide have been revealed, it gradually becomes the focus of research [11]. Animal models and molecular level studies have extensively confirmed that Irisin could enhance embryonic stem cell (ESC) neurogenesis, cell proliferation, and neural synaptic plasticity [11-14]. Importantly, Irisin has also been demonstrated to show pivotal roles in attenuating inflammation, reducing oxidative stress, and alleviating apoptosis, as well as ameliorating the function of damaged mitochondrial function [15].

Caused by brain trauma or stroke, acute brain injury (ABI) is a leading cause of death and disability, of which the incidence is increasing as the world population ages [16,17]. Brain injury will lead to common cascade of events and similar pathological processes from energy depletion to cell death, including mitochondrial dysfunction, inflammation, free-radical formation, oxidative stress, apoptosis, and so on $[18,19]$. In the exploration of repair brain damage, intervention that exerts to promote effects in the process of neuronal stem cell differentiation and maturation is worth exploring. As an emerging therapeutic strategy for brain injury, stem cell treatment is being proved to be an effective therapeutic option [20]. Interestingly, in many attempts to improve the therapeutic effect of stem cell therapy, Irisin has shown its unique advantages.

In this review, we present recently pertinent literature to describe the therapeutic potential of stem cell therapy and general roles of Irisin and display the facts of this molecule's neuroprotection in response to neurologic damage. Meanwhile, we discuss the importance of Irisin in stem cell therapy field and the possibility to be a therapeutic target for ABI.

\footnotetext{
${ }^{1}$ Department of Neurosurgery, ${ }^{3}$ Academician (Expert) Workstation of Sichuan Province, and ${ }^{4}$ Laboratory of Neurological Diseases and Brain Functions, The Affiliated Hospital of Southwest Medical University, Luzhou, China.

${ }^{2}$ Neurosurgical Clinical Research Center of Sichuan Province, Luzhou, China.
} 


\section{Stem Cell Therapy: Emerging Treatment Strategy}

The damage of functional neural cells and the deficient regeneration ability of brain are significantly characteristic pathological manifestations of ABI. Thus, to make up for the deficiency of this self-repair capacity, stem cell therapy, which dedicates to the recovery of damaged cells, has great potential.

\section{Functional cellular insults after brain injury}

In the acute phase of brain injury, the quiescently stored endogenous neural stem cells (NSCs) were activated and participated in neurorestorative process [21-23]. NSCs can differentiate into three main functional cerebral cellar lineages, including neurons, astrocytes, and oligodendrocytes. The traditional view that adult brain lacks the capability of regeneration had been broken down. The subgranular zone of the dentate gyrus in the hippocampus and the subventricular zone have been found to arise neurogenesis, for there were NSC distribution [24]. However, severe second insults would destroy all adjacent tissues, including neurons, neurogliocytes, and cerebral vascular endothelial cells, as well as the axons, fiber tracts, and supporting gliocytes, leaving the self-repair ability of brain transient and insufficient [25-28].

The limited regeneration ability of adult brain is not enough to afford to repair the damage and maintain normal function. Therefore, the exploration of an impactful treatment strategy, which can rehabilitate or even reverse the injury, is urgently needed. To achieve this purpose, stem cells, for its self-renewal property and multiply redifferential ability, have emerged as a novel alternative therapy to the cascade hypothesis of brain injury in recent years [20]. Living up to our expectations, numerous researches assessed the practicability and efficacy of application of stem cell therapies, and most of the attempts about cell therapy seem promising in distinct brain injury models [29].

\section{Brief introduction of stem cell therapy}

Investigations that aimed at improving the stem cell therapy-mediated repair process have explored many tempting treatments, involving the promotion of intrinsic neurogenesis and transplantation of exogenous NSCs [30,31].

Recently, for promoting endogenous neurogenesis, administration of various exogenous growth factors (GF) has shown evident efficacy. For example, vascular endothelial growth factor (VEGF), insulin-like growth factor-1 (IGF-1), and epidermal growth factor-like growth factor (EGF), which are powerful factors to improve neurogenesis, have always taken a place in mediating neuroplasticity and improving neurobehavioral function in various brain injury models [32-38].

Transplantation of NSCs is also in line with our expectation. In a study of brain-injured rat models, observers found that the NSCs transplanted into targeted sites of brain tissue can survive for an extended period. To select the most favorable time point and location for NSC transplantation in brain, the injured CA3 region in hippocampus and cortical white matter at 2,7 , and 14 days after injury were tested.
The NSCs which were transplanted into the interface of cortical white matter in animals at 2 days after injury could survive better, when compared to other groups. Meanwhile, the enhanced expression of mature astrocytes and oligo dendrocyte indicated that transplanted stem cells could supply the lost region-specifically functional cells [39]. In another independent study of cerebral ischemic rat models, authors observed that, after the transplantation of human NSCs (hNSCs) into the ischemic cerebral cortex, the hNSCs migrated to and survived in the lesion area, and then differentiated into functional neural lines [40]. Furthermore, Lee et al. detected the impacts of NSC transplantation on acute neural and peripheral inflammation during the hyperacute stage after hemorrhagic stroke [41]. Then they revealed that intracerebral or intravenous injection of NSCs alleviated brain edema, apoptosis, and inflammatory infiltration. As a result, they also observed the ABI animals treated with NSCs performed well in later cognitive function and motor function tests, signifying a brilliant neurologic recovery [41].

ESCs, derived from an embryo during the early blastula stage, have the potency to proliferate and differentiate into all cell lines in the development of the embryo [42]. These self-proliferating and self-sustaining population cells can provide a nearly unlimited cell source for transplantation, so it is increasingly applied in the research field of brain injury repair. In rodent animal stroke models, researchers concluded that the transplantation of ESCs would impact the neurological recovery [43-45]. On one hand, plenty of results showed that the intervention protocol of neural precursor products provided neurotrophic environment for ESCs, which are crucial for the ESCs' migration, survival, and engraftment, enhancing the amelioration of behavioral deficits and neurological deficits after brain injury [43-45], while this correlation between the efficiency of ESC therapy with the pretreatment of stem cells is a promising revelation, encouraging us for future studies. On the other hand, in different experimental brain injury animal models, researchers who successfully transplanted ESCs into brain injured animals proved that the motor function of the experimental subjects was improved [46,47].

Interestingly, because of the ability to promote the secretion of various neurogenic factors, and the effects on differentiation and maturation of stem cells as well, FNDC5/ Irisin is expected to be a novel strategy for the stem cell treatment of ABI. The role of Irisin in this regard will be described in detail later.

\section{General Description of FNDC5/Irisin}

For nearly two decades, from the controversial discovery to the confirmation of Irisins' existence, and to the exploring of its roles, Irisin gradually enters into our research vision. In this study, we introduce its origin and research process briefly.

\section{Origin of FNDC5/Irisin}

In 2002, FNDC5 was first described by two independent laboratories when learning novel fibronectin type III and peroxisomal protein $[48,49]$. The secreted portion of FNDC5 protein was discovered in 2012, which is produced by proteolytic cleavage of FNDC5. Then this protein is named by 
Boström team after Iris, the swift-footed Greek courier goddess who delivered information for the Olympian gods, underlining the significance of Irisin as a messenger to convey the metabolically related endocrine signals $[1,5,50]$.

Since 2002, Ferrer-Martínez et al. reported FNDC5 is expressed in the adult murine heart and brain [49]; then, Dun et al. evidenced the expression of Irisin in cerebellum Purkinje cells of rodents [10]. In another study, 47 different human tissues are assayed through quantitative real-time PCR. Results confirmed that brain can express FNDC5 mRNA, although the amount is low when compared to muscle [51]. Utilizing tandem mass spectrometry, circulating Irisin in human CSF is detected and quantitated by Ruan et al. [52]. Furthermore, a recent study reported that different forms of FNDC5/Irisin in immunoblots from brain homogenates are detected. Mass spectrometry analysis and immunodetection showed that immunoblot bands with differently apparent molecular weights contained the same FNDC5 peptide. The authors explained that Irisin protein may exist in different aggregation states and/or undergo posttranslational modifications [53].

\section{Link between FNDC5/Irisin and exercise}

It is well accepted that exercise is an important and necessary lifestyle, for it can help to promote healthy metabolism and protect many organ systems against a variety of disorders. These disorders include hypertension, obesity, and diabetes, which are high-risk factors for various diseases such as stroke, myocardial infarction, and other malignant conditions [53-56]. Regular exercise has long been proved to improve patients' outcomes after many neurodegenerative diseases, such as Lewy body dementia, Alzheimer's diseases (AD), and Parkinson's disease [53,57]. For patients who undergo $\mathrm{ABI}$, increased regular rehabilitation training is also correlated with their improved outcome and quality of life [58,59] (Fig. 1).

However, the potential mechanisms and the actual mediators of these protective effects of exercise are remained to be further explored. In line with this notion, exerciseinduced factors are continuously being explored. Thousands of genes and factors may be involved in this complex process [60]. FNDC5/Irisin is one of these members in a large set of exercise cohorts, mediating some of the commonly recognized benefits of exercise. As a motor gene, fndc5 encodes Irisin protein in muscle cells after exercise stimulation [61].

The concentration of Irisin in plasma differs from people with distinct age, gender, and muscle mass, as well as the level of exercise and physical status. Furthermore, it is also found that people who perform endurance exercise, also known as aerobic exercise, have high levels of serum Irisin than sedentary people [51,62]. In acute or degenerative central nervous system (CNS) diseases, a plenty of literature highlighted the neuroprotective effects of exercise mediated by FNDC5/Irisin, Irisin indicating it may play a unique role of prevention and amelioration $[5,11,53]$.

\section{Neuroprotection of FNDC5/Irisin in CNS}

Research achievements determined the existence of Irisin in CNS and laid the foundation for further research. Naturally, the exploration for the effects of this myokine in response to CNS diseases is inspired.

\section{Warrant for neurodegeneration disorders}

Since the benefits of exercise and the mediation of metabolism have been illustrated for years in neurodegeneration, Irisin acts as the pivot between metabolic effects and other neurodegenerative diseases. Recent researches highlighted the association of AD with systemic metabolic disorders such as glucose and lipid metabolic disturbance and insulin resistance [63-65]. Focusing on Irisin, it would be a new protagonist that mediates metabolism and exerts neuroprotection.

Previous studies demonstrated that FNDC5/Irisin is a myokine that promotes brain-derived neurotrophic factor (BDNF) expression in experimental mouse brain, especially in hippocampus, a brain region mainly responsible for learning and memory [6,50]. In vitro experiments by Wang et al. proved that, by regulating astrocytes, Irisin can protect

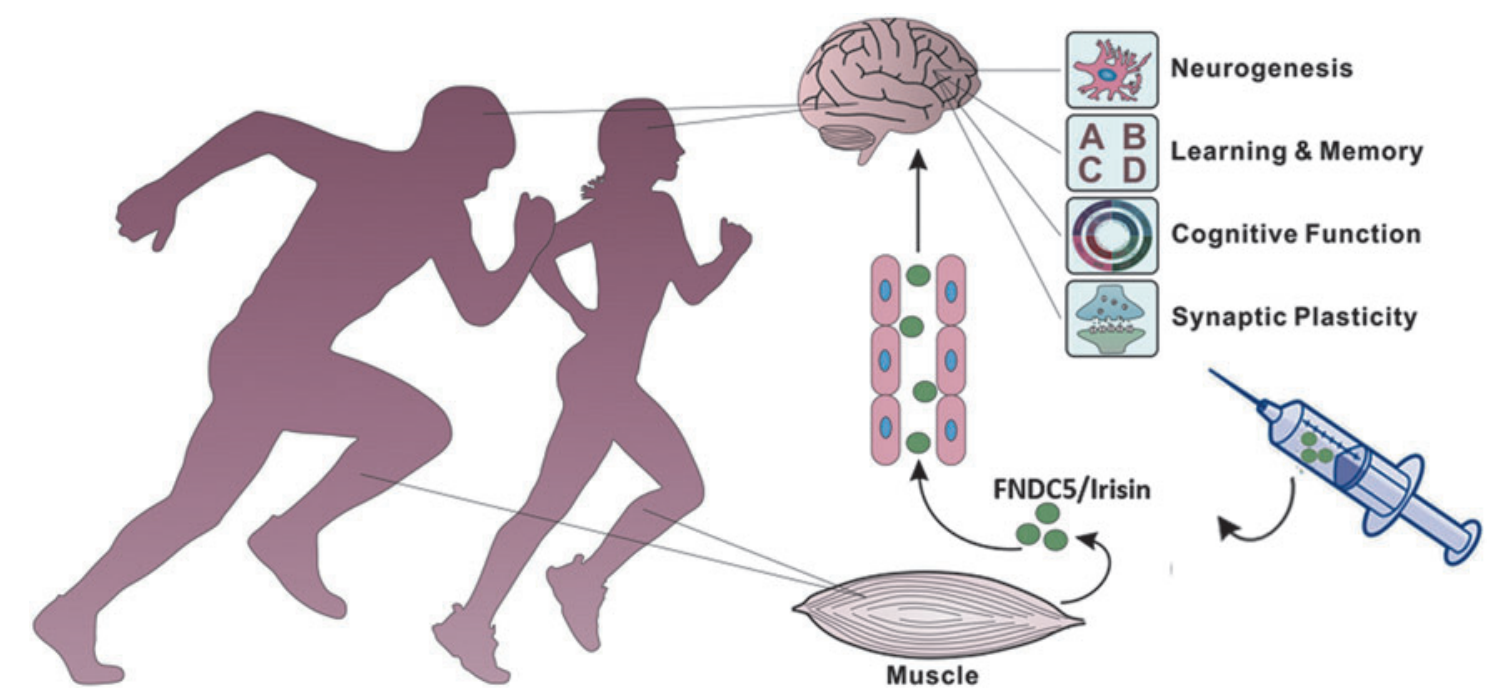

FIG. 1. Exercise-linked FNDC5/Irisin has been shown to play various significant roles in neuroprotection. 
cultured neurons against the $\beta$-amyloid toxicity [9]. In addition, the promotion of neurogenesis and neuronal cell survival contribute to the prevention of $\mathrm{AD}$ onset and the amelioration of AD pathology [65]. These results provide evidences for the Irisin peptide treatment of $\mathrm{AD}$, bringing new ideas for clinical treatment of AD. However, in the prognosis process of brain injury, there will also be impairment of learning and memory. Thus, it means that Irisin could also be potent to improve the cognitive function after ABI.

\section{Irisin in $A B I$ : initial shine}

It is in recent years that researchers gradually began to focus on the exploration of the protective effects of Irisin in ABI. In the rodent model of stroke, recent studies revealed that Irisin contributes to the neuroprotection against cerebral ischemia through various ways. Asadi et al. reported that Irisin peptide protects brain tissues against ischemic damage by reducing neural apoptosis. Meanwhile, without changing blood-brain barrier permeability, administration of Irisin showed reduction of brain edema. Moreover, by increasing the secretion of BDNF protein of cerebral cortex, it also indicated that the brain damage in experimental stroke model was attenuated [66]. Li et al. demonstrated that, by activating the Akt and ERK1/2 signaling pathways, Irisin can reduce ischemia-induced neuronal injury. They evaluated Iba-1+ microglia, MPO-1+ monocytes, and the mRNA expression of inflammation-related factors as TNF- $\alpha$ and IL-6 were diminished after Irisin treatment, placing further emphasis on the endogenous Irisin's enhancement for neuroprotective effects [67].

In oxygen-glucose deprivation (OGD) cell model, which is a commonly used in vitro model to mimic ischemic milieu, Peng et al. observed a significant decrease in the expression of both Irisin and its precursor protein FNDC5. Then they confirmed that exogenous Irisin, used as an intervention reagent, can mitigate OGD-induced neuronal injury [68].

Clinical studies also paid attention to this attractive myokine. Two independent research teams of $\mathrm{Tu}$ and $\mathrm{Wu}$ reported that the concentration of Irisin can predict functional outcomes and suggested the decreased serum levels of Irisin are associated with poor prognosis $[69,70]$. In addition, Tu et al. also demonstrated that the level of Irisin in serum is an independently powerful biological marker for predicating the risk of developing poststroke depression [71].

To date, the studies of the neuroprotective effects of Irisin in $\mathrm{ABI}$ are just emerging; however, further explorations for its potential therapeutic application are remarkably of high interest.

\section{Beneficial Effects and Underlying Mechanisms of FNDC5/Irisin}

For decades, plenty of studies provided valuable information about the properties of exercise-linked FNDC5/Irisin and opened the door to learn the potential roles and underlying mechanisms of its beneficial effects. In this section, we review essays focused on recent research developments about this myokine, which are closely related to metabolic effects and neuroprotection, and evidence to the hypothesis that Irisin could be the new protagonist in the therapeutic options of ABI.

\section{A pivot factor in metabolic regulation}

Cytokines like Irisin are key molecules for regulating energy homeostasis; thus, initial researches of Irisin mainly focused on the metabolism field [3]. As a positive result of physical exercise, the increasing circulating levels of Irisin may bring a great deal of benefits by stimulating white adipocyte browning and thermogenesis and regulating glucose [50,72].

Several studies have sought to explore the mechanisms by how this myokine is involved in modulating lipid metabolism and glucose homeostasis [72,73]. Castillo-Quan elucidated that by activating brown fat-like thermogenesis in white fat, the myokine functions in two aspects. On one hand, increase of Irisin is accompanied by white fat browning and increased oxygen and energy consumption. The reduction of body weight is our goal to fight diet-induced obesity. On the other hand, Irisin may directly improve glucose tolerance and enhance insulin sensitivity, offering new therapeutics for insulinrelated diseases [2,74]. Most of the findings illustrated the similar beneficial effects of Irisin in metabolic disease, and we present recent data to make a summary as follows:

First, the serum Irisin in obese patients or in those with type 2 diabetes (T2D) is lower than those in normal population, while the level is positively related to the amount of fatty tissue and negatively related to hyperglycemia [54,75, 76]. Second, the elevation of Irisin might cause an increase in the rest energy expenditure of obese people, and an enhancement of glucose tolerance and insulin sensitivity in diabetes mouse models [77,78]. Third, with the increase of Irisin, the significance of exercise and healthy lifestyle would be underlined again for the improvement in plasma lipid profile and glucose homeostasis in patients $[75,77,79,80]$.

Nevertheless, a few contrary points exist. Kurdiova et al. observed that the association of Irisin with obesity and metabolic phenotypes is quite controversial through two clinical studies. To test the effects of T2D, a cross-sectional study was performed in drug-naive participants. Results showed that Irisin level rose gradually with increasing obesity and worsening glucose intolerance in preclinical diabetes, while it downregulated in T2D, which pointed that Irisin might perform differently in health and metabolic disorders [81]. Another speculation of this observation might be the compensatory mechanism, while another explanation might be called "Irisin resistance," the desensitization effects of Irisin [51,82].

\section{Targeting at renovating mitochondrial homeostasis}

In the acute phase of brain injury, adequate supply of adenosine triphosphate (ATP) for repairing the damage cells is urgently needed [83]. However, damaged mitochondria could not maintain this energy demand. This imbalance in energy demand aggravated mitochondrion-centered neuronal cell death and the deleterious cascades, including electron transport chain dysfunction, ATP depletion, excessive reactive oxygen species (ROS) generation, oxidative stress damage, neuronal apoptosis, and neurogenic inflammation [84-88]. Thus, considering the significant role of mitochondria in the protection of brain damage, the search for innovative mitochondrion-targeted therapeutic regimens for $\mathrm{ABI}$ is prospective and necessary [89-91]. 
Peroxisome proliferator-activated receptor- $\gamma$ co-activator $1 \alpha(\mathrm{PGC}-1 \alpha)$ is a vital factor in the regulation of mitochondrial function, which is an upstream transcriptional regulating factor of FNDC5, and responsible for the synthesis of Irisin $[6,50]$. Through PGC- $1 \alpha$-related pathways, the serum Irisin and its expression in muscle are regulated, suggesting Irisin is a close dependent factor of PGC- $1 \alpha$ [74,92-94]. As literature reported, PGC-1 $\alpha$ shows a potent role in different aspects of regulating the mitochondrial biological activities, especially for mitochondrial biogenesis [80], while other pathological processes as oxidative stress, angiogenesis, and fiber-type switching can also be ameliorated after the regulation of PGC- $1 \alpha[80,95,96]$. Consistent with the fact that Irisin has similar effects as PGC- $1 \alpha$, significant effects of Irisin for protecting mitochondrial function and promoting mitochondrial biogenesis are reported in recent literature $[14,97,98]$. In animal and cell culture experiments, researchers demonstrated that pharmacological administration of exogenous Irisin can mitigate mitochondrial dysfunction, and as a result, ameliorating subsequent depletion of ATP. Chen et al. illustrated that Irisin could be transferred to cytoplasm by lipid raft-mediated endocytosis. And through affecting the mitochondrial uncoupling protein 2 , Irisin performed the preservation of mitochondrial function [14]. Fan et al. indicated that the Irisin protein could suppress the increase of free radical generation, inflammatory factors, and necrotic cells. Furthermore, with the treatment of Irisin, mitochondrial potential and cellular ATP would also be sustained partly through AMPK-related pathways [14,97]. By promoting mitochondrial biogenesis and inhibiting mitochondrial hypersegmentation, exogenous Irisin treatment could increase the mitochondrial contents; thus, the overconsumed ATP would be compensated [98]. Thereby, cascade reaction simultaneously activated by mitochondrion-centered disorders would also be alleviated by mitigating the impairment of mitochondrial function and integrity $[87,88]$.

Notably, since brain is an organ that is particularly sensitive to oxygen, cerebral ischemia or hypoxia can contribute to poor prognosis. All of these points related to mitochondria are crucial in most of the acute pathological process, especially in acute neurovascular injury. Thus, on the basis of Irisin's protective roles on mitochondrial function, we can conclude the importance of this protein's neuroprotection in acute neural trauma.

\section{Potent neurotrophin for normal brain function maintenance}

Among multifarious neurotrophic agents, BDNF has always been the most widely studied focus [99]. This shining star performs neuroprotective roles through various aspects, including facilitating neural regeneration and adult neurogenesis, mediating synaptogenesis, and synaptic plasticity, as well as enhancing cell survival and reducing apoptosis [100-103]. Thereby, BDNF is effective in improving the recovery of neurologic functions and abilities associated with memory, learning, and perceptual motion. A growing body of evidences demonstrated that, among various types of neurotrophic candidates, BDNF might be a particularly good participant to perform survival-promoting effects in cerebral lesions [101,104,105].
Clinically, researchers observed that BDNF levels could be a biomarker for predicting mortality and the outcome after cerebral injury [106-108]. In animal studies, the therapeutic effects of BDNF also make researchers exciting. Schäbitz et al. proved that after small cortical ischemia, BDNF intervention could enhance functional motor recovery. At the cellular level, the inducing of widespread neuronal remodeling and reducing of postischemic astrogliosis were observed [101]. Even in the research field of stem cell therapy, BDNF is also validated to participate in the process of stem cell proliferation, differentiation, and migration [22].

Of these, it is interesting to note that this potent neurotrophic factor is mediated by Irisin through a PGC- $1 \alpha /$ FNDC5 pathway, and exercise or forced way to stimulate the expression of serum Irisin could induce the elevation of BDNF in the hippocampus [109]. Meanwhile, the downregulated expression of BDNF could be observed when using siRNA to manipulate fndc 5 expression in cortical neuron [6]. Thus, as a downstream factor, BDNF enhanced the neuroprotective effects of Irisin, highlighted Irisin's pivotal role in early cerebral injury cascade again, and, to some extent, explained a part of its mechanism.

\section{Response to commonly shared pathological process in $\mathrm{ABI}$}

In various cases of $\mathrm{ABI}$ involving traumatic brain injury, ischemic stroke, and hemorrhagic stroke, some pathological changes are overlapped [110]. Therefore, it is reasonable for researchers to illustrate the underlying mechanisms of Irisin from some common pathophysiologic process in secondary brain injury cascade. Consistent with our perspective, a great deal of researchers demonstrated the protective potential of Irisin in inflammation, oxidative stress, apoptosis, neurodegeneration, and other pathological disorders, which are the dominant deterioration in the acute phase of brain damage [15].

Neuroinflammation and oxidative stress are critically detrimental conditions in neural lesion [20,110]. Studies reported the anti-inflammatory and antioxidative latent properties of Irisin $[67,68]$. In the middle cerebral artery occlusion animal models and the OGD-cultured PC12 neuronal cells, investigators observed that exogenous Irisin injection could suppress inflammation and oxidative stress. As is known, microglia and neutrophils are important neuroinflammatory cells in ABI. After intervention of Irisin, the activation of Iba- $1^{+}$and MPO- $1^{+}$cells, which are the markers of microglia and neutrophils, is inhibited. The mRNA expression of proinflammatory cytokines involving IL-6 and TNF- $\alpha$ are all reduced. Meanwhile, the levels of some oxidative stress parameters involving nitrotyrosine, superoxide anion, and 4-hydroxynonenal decreased after Irisin administration [67]. Further with this, researchers expounded that Irisin treatment reversed OGD-induced inflammation and oxidative stress through ROS-NLRP3 pathway [68]. A new finding reported that optimum dose of Irisin attenuated ischemia-induced apoptotic cell death with the upregulation of Bcl-2 and downregulation of Bax, which are two important gene products in the intrinsic apoptosis regulation system [66].

In addition, cumulative evidences from other investigations demonstrated that Irisin treatment ameliorated inflammation, oxidative stress, and apoptosis in different disease 
models, including myocardial infarction [111,112], pulmonary injury [14,113], hepatic ischemia [98], pancreatic dysfunction [114], kidney disease [115], and atherosclerosis [116].

Moreover, adaptive autophagy, as an evolutionarily selfprotective mechanism, serves prominent protective effects in acute cerebral injury [117]. It is documented that autophagy system is activated during early brain injury (EBI), and the promotion of neuronal autophagy may effectively attenuate neurologic deficit [118]. In animal experiments, Irisin is also well confirmed to show its critical benefits for cardiomyocyte survival by inducing protective autophagy $[119,120]$.

To sum up, given the multiple effects of Irisin, diseases with such evidently common pathologies in acute phase can be targeted by Irisin treatment, and all evidences suggest that investigation in this field will remarkably make sense.

\section{Promising option: FNDC5/Irisin and stem cell therapy}

In the process of neuronal differentiation and maturation, FNDC5/Irisin plays a promoting effect. At the genetic level, studies have confirmed that fndc5 gene is of importance in the germination and development of the central nervous system. Hashemi et al. constructed a mouse ESC line, which could stably express shRNA to knock out the fndc5 gene, and then used these cell lines to knock down the fndc5 gene in the process of mouse ESC neural differentiation, both in the stages of neural progenitor (NP) formation and post-NP formation. In addition to fndc5 gene knockdown significantly cutting down the expression of NPs in the NP formation stage, their experimental results also indicated that decreased fndc5 expression could reduce the mature neuronal markers, affecting the maturation and differentiation of neurons and astrocytes [121] (Fig. 2).

Recently, this work team used retinoic acid (RA) to force the overexpression of FNDC5, and they observed that markers of neuronal precursor and mature neuron significantly increased. These results indicated that the enhanced expression of FNDC5 could facilitate the maturation and differentiation process of primary mouse embryonic cortical neurons [13]. Meanwhile, another study expanded the information on the effects of fndc5 during neural differentiation and maturation. In all cell types, included human ESCs, neural cells, and rosette structures, three fndc5 subtypes contiguously increase during the neural differentiation process, while the highest expression level of fndc5 isoforms is in neural cells [12].
To observe whether Irisin could directly regulate the neurogenesis process, researchers as Moon et al. conducted a study in vitro. Compared with the control group, the pharmacological concentrations of Irisin treatment could enhance cell proliferation, while physiological concentrations of Irisin showed no obvious effect [122].

The above evidences suggest that fndc5 gene and its coding product Irisin peptide are of significance in neural tube germination and development. Given the remarkable properties of FNDC5/Irisin in the process of ESC differentiation and maturation, and the confirmed ability for mediating BDNF, we can make a well-founded hypothesis that Irisin could be a pivot candidate for stem cell therapy. Future studies are required to confirm the observed protective effects of Irisin, and to reveal the precise therapeutic relevance between Irisin and stem cell treatment, as well as the related potential mechanisms.

\section{Perspectives}

Plenty of clinical and animal experiment data linked the health benefits of exercise with many diseases [53-56,59, $81,123]$. However, many patients, who suffered brain injury or other poor conditions that made them disabled, would be prevented from taking part in regular physical activities. Efforts to explore and develop alternative approaches of these exercise-linked beneficial effects may benefit those populations. In this reality, the potent neuroprotective roles endow Irisin the ability to execute the task, and Irisin intervention would be a meaningful attempt for those who cannot enjoy the benefits of exercise.

Clinically, we usually find the patients those suffered ABI caused by different reasons are commonly affected by some co-ailments, which are related to metabolic dysfunction [124-127]. However, in most of animal model studies, researchers predominantly choose young and healthy animals. However, age and other comorbidities would lead to unsuccessful clinical translation. Fortunately, given the potent properties of Irisin in metabolic disorders, Irisin peptide treatment makes it possible to take comorbidities into account. Thus, this intervention of Irisin would be more promising and meaningful in clinical translation.

Given the pluripotent regeneration capacity of the stem cells, the related stem cell therapy has been explored in depth. Stem cell transplantation has clinical application value for brain injury; however, many factors affect the efficacy of stem cell therapy, of which, mitochondrial
FIG. 2. FNDC5/Irisin would be a promising option for stem cell therapy.

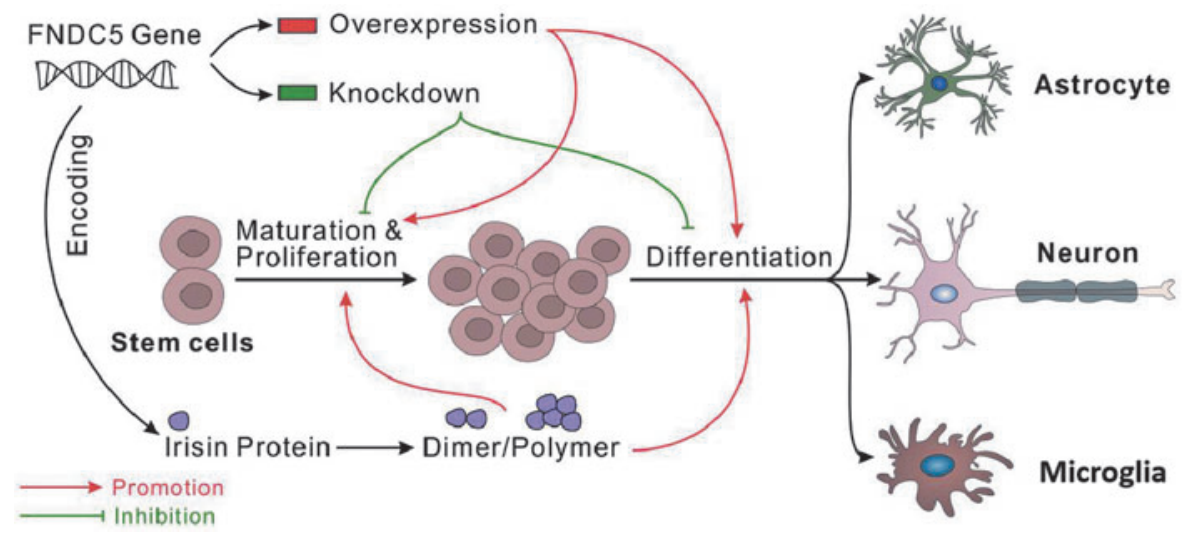


dysfunction is a factor that should not be ignored, for the mitochondrion acts as a central regulator of stem cell survival [128,129]. On the other hand, metabolism is also closely related to the survival and therapeutic effects of stem cell therapy [130-132]. To ensure successful clinical transformation, we must consider thoroughly without neglecting these metabolic factors. Comprehensive treatment strategy is necessary guarantee for improving the possibility of clinical transformation, and any therapeutic administration should take these points into account. Notably, while regulating metabolic disorders, Irisin could stabilize mitochondrial function as well; thus, the effects of the stem cell treatment can be better enhanced by Irisin intervention.

\section{Conclusion}

The treatment strategies for brain injury have been explored for decades; however, the transformation of clinical effect is not very satisfactory. To achieve more effective and more scientific clinical transformation, new alternative treatment strategies are still needed to be explored. Currently, stem cell therapy would be a promising approach to improve behavioral dysfunction and increase recovery after brain injury, while Irisin peptide may be a novel and ideal target to enhance its curative effects from various aspects. Remarkably, the interesting journey of exploring the potential mechanisms has just begun. To uncover the mechanisms of potently protective effects of Irisin, future studies about the precise therapeutic relevance are required.

\section{Author Disclosure Statement}

No competing financial interests exist.

\section{Funding Information}

This work was supported by the National Natural Science Foundation of China (81771278, 81801176, and 81971132), Sichuan Science and Technology Program (2018RZ0090, 2019JDTD0004 and 19MZGC0003), and Luzhou Science and Technology Program (2016LZXNYD-Z02, 2017-R-74, 2018-JYJ-42).

\section{References}

1. Farmer SR. (2019). Boning up on irisin. N Engl J Med 380:1480-1482.

2. Wu J, P Bostrom, LM Sparks, L Ye, JH Choi, AH Giang, M Khandekar, KA Virtanen, P Nuutila, et al. (2012). Beige adipocytes are a distinct type of thermogenic fat cell in mouse and human. Cell 150:366-376.

3. Boström PA and JM Fernández-Real. (2013). Irisin, the metabolic syndrome and follistatin in humans. Nat Rev Endocrinol 10:11-12.

4. Villarroya F. (2012). Irisin, turning up the heat. Cell Metab 15:277-278.

5. Young MF, S Valaris and CD Wrann. (2019). A role for FNDC5/Irisin in the beneficial effects of exercise on the brain and in neurodegenerative diseases. Prog Cardiovasc Dis 62:172-178.

6. Wrann CD, JP White, J Salogiannnis, D LaznikBogoslavski, J Wu, D Ma, JD Lin, ME Greenberg and BM Spiegelman. (2013). Exercise induces hippocampal BDNF through a PGC-1alpha/FNDC5 pathway. Cell Metab 18: 649-659.

7. Varela-Rodriguez BM, L Pena-Bello, P Juiz-Valina, B Vidal-Bretal, F Cordido and S Sangiao-Alvarellos. (2016). FNDC5 expression and circulating irisin levels are modified by diet and hormonal conditions in hypothalamus, adipose tissue and muscle. Sci Rep 6:29898.

8. Piya MK, AL Harte, K Sivakumar, G Tripathi, PD Voyias, S James, S Sabico, NM Al-Daghri, P Saravanan, et al. (2014). The identification of irisin in human cerebrospinal fluid: influence of adiposity, metabolic markers, and gestational diabetes. Am J Physiol Endocrinol Metab 306: E512-E518.

9. Wang K, H Li, H Wang, JH Wang, F Song and Y Sun. (2018). Irisin exerts neuroprotective effects on cultured neurons by regulating astrocytes. Mediators Inflamm 2018:9070341.

10. Dun SL, RM Lyu, YH Chen, JK Chang, JJ Luo and NJ Dun. (2013). Irisin-immunoreactivity in neural and nonneural cells of the rodent. Neuroscience 240:155-162.

11. Tari AR, CS Norevik, NR Scrimgeour, A KobroFlatmoen, J Storm-Mathisen, LH Bergersen, CD Wrann, G Selbaek, M Kivipelto, JBN Moreira and U Wisloff. (2019). Are the neuroprotective effects of exercise training systemically mediated? Prog Cardiovasc Dis 62: 94-101.

12. Ghahrizjani FA, K Ghaedi, A Salamian, S Tanhaei, AS Nejati, H Salehi, M Nabiuni, H Baharvand and MH Nasr-Esfahani. (2015). Enhanced expression of FNDC5 in human embryonic stem cell-derived neural cells along with relevant embryonic neural tissues. Gene 557: 123-129.

13. Forouzanfar M, F Rabiee, K Ghaedi, S Beheshti, S Tanhaei, A Shoaraye Nejati, M Jodeiri Farshbaf, $\mathrm{H}$ Baharvand and MH Nasr-Esfahani. (2015). Fndc5 overexpression facilitated neural differentiation of mouse embryonic stem cells. Cell Biol Int 39:629-637.

14. Chen K, Z Xu, Y Liu, Z Wang, Y Li, X Xu, C Chen, T Xia, Q Liao, et al. (2017). Irisin protects mitochondria function during pulmonary ischemia/reperfusion injury. Sci Transl Med 9. DOI: 10.1126/scitranslmed.aao6298.

15. Askari H, SF Rajani, M Poorebrahim, H Haghi-Aminjan, E Raeis-Abdollahi and M Abdollahi. (2018). A glance at the therapeutic potential of irisin against diseases involving inflammation, oxidative stress, and apoptosis: an introductory review. Pharmacol Res 129:44-55.

16. GBD 2016 Traumatic Brain Injury and Spinal Cord Injury Collaborators. (2019). Global, regional, and national burden of traumatic brain injury and spinal cord injury, 1990-2016: a systematic analysis for the Global Burden of Disease Study 2016. Lancet Neurol 18:56-87.

17. Joy MT, E Ben Assayag, D Shabashov-Stone, S LirazZaltsman, J Mazzitelli, M Arenas, N Abduljawad, E Kliper, AD Korczyn, et al. (2019). CCR5 is a therapeutic target for recovery after stroke and traumatic brain injury. Cell 176:1143-1157 e13.

18. Dirnagl U, C Iadecola and MA Moskowitz. (1999). Pathobiology of ischaemic stroke: an integrated view. Trends Neurosci 22:391-397.

19. Corps KN, TL Roth and DB McGavern. (2015). Inflammation and neuroprotection in traumatic brain injury. JAMA Neurol 72:355-362.

20. Reis C, V Gospodarev, H Reis, M Wilkinson, J Gaio, C Araujo, S Chen and JH Zhang. (2017). Traumatic brain 
injury and stem cell: pathophysiology and update on recent treatment modalities. Stem Cells Int 2017:6392592.

21. Llorens-Bobadilla E, S Zhao, A Baser, G Saiz-Castro, K Zwadlo and A Martin-Villalba. (2015). Single-cell transcriptomics reveals a population of dormant neural stem cells that become activated upon brain injury. Cell Stem Cell 17:329-340.

22. $\mathrm{Hu} \mathrm{W}, \mathrm{W}-\mathrm{D}$ Lee, K-C Wang, Y-F Tsai, P-C Chou, L-K Tsai and C-L Chien. (2016). Subarachnoid hemorrhage promotes proliferation, differentiation, and migration of neural stem cells via BDNF upregulation. Plos One 11: e0165460.

23. Dillen Y, H Kemps, P Gervois, E Wolfs and A Bronckaers. (2019). Adult neurogenesis in the subventricular zone and its regulation after ischemic stroke: implications for therapeutic approaches. Transl Stroke Res 11:60-79.

24. Ming GL and H Song. (2011). Adult neurogenesis in the mammalian brain: significant answers and significant questions. Neuron 70:687-702.

25. Xiong Y, A Mahmood and M Chopp. (2013). Animal models of traumatic brain injury. Nat Rev Neurosci 14: 128-142.

26. Huang L and L Zhang. (2019). Neural stem cell therapies and hypoxic-ischemic brain injury. Prog Neurobiol 173: $1-17$.

27. Wu Y, J Peng, J Pang, X Sun and Y Jiang. (2017). Potential mechanisms of white matter injury in the acute phase of experimental subarachnoid haemorrhage. Brain 140:e36.

28. Titomanlio L, A Kavelaars, J Dalous, S Mani, V El Ghouzzi, C Heijnen, O Baud and P Gressens. (2011). Stem cell therapy for neonatal brain injury: perspectives and challenges. Ann Neurol 70:698-712.

29. Pluchino S, L Zanotti, B Rossi, E Brambilla, L Ottoboni, G Salani, M Martinello, A Cattalini, A Bergami, et al. (2005). Neurosphere-derived multipotent precursors promote neuroprotection by an immunomodulatory mechanism. Nature 436:266-271.

30. Sarmah D, H Kaur, J Saraf, K Pravalika, A Goswami, K Kalia, A Borah, X Wang, KR Dave, DR Yavagal and $\mathrm{P}$ Bhattacharya. (2018). Getting closer to an effective intervention of ischemic stroke: the big promise of stem cell. Transl Stroke Res 9:356-374.

31. Takagi T, S Yoshimura, R Sakuma, A Nakano-Doi, $\mathrm{T}$ Matsuyama and T Nakagomi. (2017). Novel regenerative therapies based on regionally induced multipotent stem cells in post-stroke brains: their origin, characterization, and perspective. Transl Stroke Res 8:515-528.

32. Sugiura S, K Kitagawa, S Tanaka, K Todo, E OmuraMatsuoka, T Sasaki, T Mabuchi, K Matsushita, Y Yagita and M Hori. (2005). Adenovirus-mediated gene transfer of heparin-binding epidermal growth factor-like growth factor enhances neurogenesis and angiogenesis after focal cerebral ischemia in rats. Stroke 36:859-864.

33. Carlson SW and KE Saatman. (2018). Central infusion of insulin-like growth factor-1 increases hippocampal neurogenesis and improves neurobehavioral function after traumatic brain injury. J Neurotrauma 35:1467-1480.

34. Carlson SW, SK Madathil, DM Sama, X Gao, J Chen and KE Saatman. (2014). Conditional overexpression of insulin-like growth factor-1 enhances hippocampal neurogenesis and restores immature neuron dendritic processes after traumatic brain injury. J Neuropathol Exp Neurol 73:734-746.
35. Lu KT, CL Sun, PY Wo, HH Yen, TH Tang, MC Ng, ML Huang and YL Yang. (2011). Hippocampal neurogenesis after traumatic brain injury is mediated by vascular endothelial growth factor receptor- 2 and the Raf/MEK/ERK cascade. J Neurotrauma 28:441-450.

36. Lee C and DV Agoston. (2010). Vascular endothelial growth factor is involved in mediating increased de novo hippocampal neurogenesis in response to traumatic brain injury. J Neurotrauma 27:541-553.

37. Jin K, Y Sun, L Xie, J Childs, XO Mao and DA Greenberg. (2004). Post-ischemic administration of heparin-binding epidermal growth factor-like growth factor (HB-EGF) reduces infarct size and modifies neurogenesis after focal cerebral ischemia in the rat. J Cereb Blood Flow Metab 24:399-408.

38. Thau-Zuchman O, E Shohami, AG Alexandrovich and RR Leker. (2010). Vascular endothelial growth factor increases neurogenesis after traumatic brain injury. J Cereb Blood Flow Metab 30:1008-1016.

39. Sun D, M Gugliotta, A Rolfe, W Reid, AR McQuiston, W Hu and H Young. (2011). Sustained survival and maturation of adult neural stem/progenitor cells after transplantation into the injured brain. J Neurotrauma 28: 961-972.

40. Kelly S, TM Bliss, AK Shah, GH Sun, M Ma, WC Foo, J Masel, MA Yenari, IL Weissman, et al. (2004). Transplanted human fetal neural stem cells survive, migrate, and differentiate in ischemic rat cerebral cortex. Proc Natl Acad Sci U S A 101:11839-11844.

41. Lee ST, K Chu, KH Jung, SJ Kim, DH Kim, KM Kang, NH Hong, JH Kim, JJ Ban, et al. (2008). Anti-inflammatory mechanism of intravascular neural stem cell transplantation in haemorrhagic stroke. Brain 131:616-629.

42. Evans M. (2011). Discovering pluripotency: 30 years of mouse embryonic stem cells. Nat Rev Mol Cell Biol 12: 680-686.

43. Drury-Stewart D, M Song, O Mohamad, Y Guo, X Gu, D Chen and L Wei. (2013). Highly efficient differentiation of neural precursors from human embryonic stem cells and benefits of transplantation after ischemic stroke in mice. Stem Cell Res Ther 4:93.

44. Yang T, KS Tsang, WS Poon and HK Ng. (2009). Neurotrophism of bone marrow stromal cells to embryonic stem cells: noncontact induction and transplantation to a mouse ischemic stroke model. Cell Transplant 18: 391-404.

45. Fong SP, KS Tsang, AB Chan, G Lu, WS Poon, K Li, LW Baum and HK Ng. (2007). Trophism of neural progenitor cells to embryonic stem cells: neural induction and transplantation in a mouse ischemic stroke model. J Neurosci Res 85:1851-1862.

46. Peruzzaro ST, J Gallagher, J Dunkerson, S Fluharty, D Mudd, MR Hoane and JS Smith. (2013). The impact of enriched environment and transplantation of murine cortical embryonic stem cells on recovery from controlled cortical contusion injury. Restor Neurol Neurosci 31:431450.

47. Ikeda R, MS Kurokawa, S Chiba, H Yoshikawa, M Ide, M Tadokoro, S Nito, N Nakatsuji, Y Kondoh, et al. (2005). Transplantation of neural cells derived from retinoic acid-treated cynomolgus monkey embryonic stem cells successfully improved motor function of hemiplegic mice with experimental brain injury. Neurobiol Dis 20: $38-48$. 
48. Teufel A, N Malik, M Mukhopadhyay and H Westphal. (2002). Frcp1 and Frcp2, two novel fibronectin type III repeat containing genes. Gene 297:79-83.

49. Ferrer-Martínez A, P Ruiz-Lozano and KR Chien. (2002). Mouse PeP: a novel peroxisomal protein linked to myoblast differentiation and development. Dev Dyn 224: 154-167.

50. Bostrom P, J Wu, MP Jedrychowski, A Korde, L Ye, JC Lo, KA Rasbach, EA Bostrom, JH Choi, et al. (2012). A PGC1-alpha-dependent myokine that drives brown-fatlike development of white fat and thermogenesis. Nature 481:463-468.

51. Huh JY, G Panagiotou, V Mougios, M Brinkoetter, MT Vamvini, BE Schneider and CS Mantzoros. (2012). FNDC5 and irisin in humans: I. Predictors of circulating concentrations in serum and plasma and II. mRNA expression and circulating concentrations in response to weight loss and exercise. Metabolism 61:1725-1738.

52. Ruan Q, L Zhang, J Ruan, X Zhang, J Chen, C Ma and $\mathrm{Z} \mathrm{Yu}$. (2018). Detection and quantitation of irisin in human cerebrospinal fluid by tandem mass spectrometry. Peptides 103:60-64.

53. Lourenco MV, RL Frozza, GB de Freitas, H Zhang, GC Kincheski, FC Ribeiro, RA Goncalves, JR Clarke, D Beckman, et al. (2019). Exercise-linked FNDC5/irisin rescues synaptic plasticity and memory defects in Alzheimer's models. Nat Med 25:165-175.

54. Peterson JM, R Mart and CE Bond. (2014). Effect of obesity and exercise on the expression of the novel myokines, Myonectin and Fibronectin type III domain containing 5. PeerJ 2:e605.

55. Raina Elley C and B Arroll. (2005). Refining the exercise prescription for hypertension. Lancet 366:1248-1249.

56. Khan KM, AM Thompson, SN Blair, JF Sallis, KE Powell, FC Bull and AE Bauman. (2012). Sport and exercise as contributors to the health of nations. Lancet 380:59-64.

57. Bailey DM, TA Calverley and R Corkill. (2019). Drugs for dementia: exercise is medicine. BMJ 364:k5438.

58. Tan CO, WP Meehan, 3rd, GL Iverson and JA Taylor. (2014). Cerebrovascular regulation, exercise, and mild traumatic brain injury. Neurology 83:1665-1672.

59. Dobkin BH. (2008). Training and exercise to drive poststroke recovery. Nat Clin Pract Neurol 4:76-85.

60. Kim S, JY Choi, S Moon, DH Park, HB Kwak and JH Kang. (2019). Roles of myokines in exercise-induced improvement of neuropsychiatric function. Pflugers Arch 471:491-505.

61. Timmons JA, K Baar, PK Davidsen and PJ Atherton. (2012). Is irisin a human exercise gene? Nature 488: E9-E10.

62. Jedrychowski MP, CD Wrann, JA Paulo, KK Gerber, J Szpyt, MM Robinson, KS Nair, SP Gygi and BM Spiegelman. (2015). Detection and quantitation of circulating human irisin by tandem mass spectrometry. Cell Metab 22:734-740.

63. Murphy MP. (2018). Amyloid-beta solubility in the treatment of Alzheimer's disease. N Engl J Med 378:391392.

64. Barnett R. (2019). Alzheimer's disease. Lancet 393:1589.

65. Kim OY and J Song. (2018). The role of irisin in Alzheimer's disease. J Clin Med 7:407.

66. Asadi Y, F Gorjipour, S Behrouzifar and A Vakili. (2018). Irisin peptide protects brain against ischemic injury through reducing apoptosis and enhancing BDNF in a rodent model of stroke. Neurochem Res 43:1549-1560.

67. Li DJ, YH Li, HB Yuan, LF Qu and P Wang. (2017). The novel exercise-induced hormone irisin protects against neuronal injury via activation of the Akt and ERK1/2 signaling pathways and contributes to the neuroprotection of physical exercise in cerebral ischemia. Metabolism 68: 31-42.

68. Peng J, X Deng, W Huang, JH Yu, JX Wang, JP Wang, SB Yang, X Liu, L Wang, et al. (2017). Irisin protects against neuronal injury induced by oxygen-glucose deprivation in part depends on the inhibition of ROS-NLRP3 inflammatory signaling pathway. Mol Immunol 91:185194.

69. Tu WJ, HC Qiu, JL Cao, Q Liu, XW Zeng and JZ Zhao. (2018). Decreased concentration of irisin is associated with poor functional outcome in ischemic stroke. Neurotherapeutics 15:1158-1167.

70. Wu H, P Guo, Z Jin, X Li, X Yang, C Tang, Y Wang and J Ke. (2019). Serum levels of irisin predict short-term outcomes in ischemic stroke. Cytokine 122:154303.

71. Tu WJ, HC Qiu, Q Liu, X Li, JZ Zhao and X Zeng. (2018). Decreased level of irisin, a skeletal muscle cellderived myokine, is associated with post-stroke depression in the ischemic stroke population. $\mathrm{J}$ Neuroinflammation $15: 133$.

72. Hocking S, D Samocha-Bonet, KL Milner, JR Greenfield and DJ Chisholm. (2013). Adiposity and insulin resistance in humans: the role of the different tissue and cellular lipid depots. Endocr Rev 34:463-500.

73. Perakakis N, GA Triantafyllou, JM Fernandez-Real, JY Huh, KH Park, J Seufert and CS Mantzoros. (2017). Physiology and role of irisin in glucose homeostasis. Nat Rev Endocrinol 13:324-337.

74. Castillo-Quan JI. (2012). From white to brown fat through the PGC-1alpha-dependent myokine irisin: implications for diabetes and obesity. Dis Model Mech 5:293-295.

75. Moreno-Navarrete JM, F Ortega, M Serrano, E Guerra, G Pardo, F Tinahones, W Ricart and JM Fernandez-Real. (2013). Irisin is expressed and produced by human muscle and adipose tissue in association with obesity and insulin resistance. J Clin Endocrinol Metab 98:E769-E778.

76. Liu JJ, MD Wong, WC Toy, CS Tan, S Liu, XW Ng, S Tavintharan, CF Sum and SC Lim. (2013). Lower circulating irisin is associated with type 2 diabetes mellitus. J Diabetes Complications 27:365-369.

77. Stengel A, T Hofmann, M Goebel-Stengel, U Elbelt, P Kobelt and BF Klapp. (2013). Circulating levels of irisin in patients with anorexia nervosa and different stages of obesity - correlation with body mass index. Peptides 39: 125-130.

78. Zhu G, J Wang, M Song, F Zhou, D Fu, G Ruan, X Zhu, Y Bai, L Huang, et al. (2016). Irisin increased the number and improved the function of endothelial progenitor cells in diabetes mellitus mice. J Cardiovasc Pharmacol 68: 67-73.

79. Bluher S, G Panagiotou, D Petroff, J Markert, A Wagner, T Klemm, A Filippaios, A Keller and CS Mantzoros. (2014). Effects of a 1-year exercise and lifestyle intervention on irisin, adipokines, and inflammatory markers in obese children. Obesity (Silver Spring) 22:1701-1708

80. Fernandez-Marcos PJ and J Auwerx. (2011). Regulation of PGC-1alpha, a nodal regulator of mitochondrial biogenesis. Am J Clin Nutr 93:884S-890S. 
81. Kurdiova T, M Balaz, M Vician, D Maderova, M Vlcek, L Valkovic, M Srbecky, R Imrich, O Kyselovicova, et al. (2014). Effects of obesity, diabetes and exercise on Fndc5 gene expression and irisin release in human skeletal muscle and adipose tissue: in vivo and in vitro studies. J Physiol 592:1091-1107.

82. Polyzos SA, J Kountouras, K Shields and CS Mantzoros. (2013). Irisin: a renaissance in metabolism? Metabolism 62:1037-1044.

83. Hagberg H, C Mallard, CI Rousset and C Thornton. (2014). Mitochondria: hub of injury responses in the developing brain. Lancet Neurol 13:217-232.

84. Yang J-L, S Mukda and S-D Chen. (2018). Diverse roles of mitochondria in ischemic stroke. Redox Biol 16:263275.

85. Chou SHY, J Lan, E Esposito, M Ning, L Balaj, X Ji, EH Lo and K Hayakawa. (2017). Extracellular mitochondria in cerebrospinal fluid and neurological recovery after subarachnoid hemorrhage. Stroke 48:2231-2237.

86. Bolanos JP, MA Moro, I Lizasoain and A Almeida. (2009). Mitochondria and reactive oxygen and nitrogen species in neurological disorders and stroke: therapeutic implications. Adv Drug Deliv Rev 61:1299-1315.

87. Cheng G, R-H Kong, L-M Zhang and J-N Zhang. (2012). Mitochondria in traumatic brain injury and mitochondrialtargeted multipotential therapeutic strategies. Br J Pharmacol 167:699-719.

88. Sims NR and H Muyderman. (2010). Mitochondria, oxidative metabolism and cell death in stroke. Biochim Biophys Acta 1802:80-91.

89. Hayakawa K, E Esposito, X Wang, Y Terasaki, Y Liu, C Xing, X Ji and EH Lo. (2016). Transfer of mitochondria from astrocytes to neurons after stroke. Nature 535:551555.

90. Ji J, AE Kline, A Amoscato, AK Samhan-Arias, LJ Sparvero, VA Tyurin, YY Tyurina, B Fink, MD Manole, et al. (2012). Lipidomics identifies cardiolipin oxidation as a mitochondrial target for redox therapy of brain injury. Nat Neurosci 15:1407-1413.

91. Mo J, B Enkhjargal, ZD Travis, K Zhou, P Wu, G Zhang, Q Zhu, T Zhang, J Peng, et al. (2019). AVE 0991 attenuates oxidative stress and neuronal apoptosis via Mas/ PKA/CREB/UCP-2 pathway after subarachnoid hemorrhage in rats. Redox Biol 20:75-86.

92. Kazeminasab F, SM Marandi, K Ghaedi, Z Safaeinejad, F Esfarjani and MH Nasr-Esfahani. (2018). A comparative study on the effects of high-fat diet and endurance training on the PGC-1alpha-FNDC5/irisin pathway in obese and nonobese male C57BL/6 mice. Appl Physiol Nutr Metab 43:651-662.

93. Ye X, Y Shen, C Ni, J Ye, Y Xin, W Zhang and Y Ren. (2019). Irisin reverses insulin resistance in C2C12 cells via the p38-MAPK-PGC-1alpha pathway. Peptides 119: 170120.

94. Zhou Q, K Chen, P Liu, Y Gao, D Zou, H Deng, Y Huang, Q Zhang, J Zhu and M Mi. (2015). Dihydromyricetin stimulates irisin secretion partially via the PGC-1alpha pathway. Mol Cell Endocrinol 412:349-357.

95. Wu Z, P Puigserver, U Andersson, C Zhang, G Adelmant, V Mootha, A Troy, S Cinti, B Lowell, RC Scarpulla and BM Spiegelman. (1999). Mechanisms controlling mitochondrial biogenesis and respiration through the thermogenic coactivator PGC-1. Cell 98:115-124.
96. Handschin C and BM Spiegelman. (2008). The role of exercise and PGC1alpha in inflammation and chronic disease. Nature 454:463-469.

97. Fan J, Q Zhu, Z Wu, J Ding, S Qin, H Liu and P Miao. (2019). Protective effects of irisin on hypoxiareoxygenation injury in hyperglycemia-treated cardiomyocytes: role of AMPK pathway and mitochondrial protection. J Cell Physiol 235:1165-1174.

98. Bi J, J Zhang, Y Ren, Z Du, Q Li, Y Wang, S Wei, L Yang, J Zhang, et al. (2019). Irisin alleviates liver ischemia-reperfusion injury by inhibiting excessive mitochondrial fission, promoting mitochondrial biogenesis and decreasing oxidative stress. Redox Biol 20:296-306.

99. Choi SH, E Bylykbashi, ZK Chatila, SW Lee, B Pulli, GD Clemenson, E Kim, A Rompala, MK Oram, et al. (2018). Combined adult neurogenesis and BDNF mimic exercise effects on cognition in an Alzheimer's mouse model. Science 361. DOI: 10.1126/science.aan8821.

100. Clarkson AN, JJ Overman, S Zhong, R Mueller, G Lynch and ST Carmichael. (2011). AMPA receptor-induced local brain-derived neurotrophic factor signaling mediates motor recovery after stroke. J Neurosci 31:3766-3775.

101. Schabitz WR, C Berger, R Kollmar, M Seitz, E Tanay, M Kiessling, S Schwab and C Sommer. (2004). Effect of brain-derived neurotrophic factor treatment and forced arm use on functional motor recovery after small cortical ischemia. Stroke 35:992-997.

102. Schabitz WR, T Steigleder, CM Cooper-Kuhn, S Schwab, C Sommer, A Schneider and HG Kuhn. (2007). Intravenous brain-derived neurotrophic factor enhances poststroke sensorimotor recovery and stimulates neurogenesis. Stroke 38:2165-2172.

103. Guan J, W Tong, W Ding, S Du, Z Xiao, Q Han, Z Zhu, $X$ Bao, X Shi, et al. (2012). Neuronal regeneration and protection by collagen-binding BDNF in the rat middle cerebral artery occlusion model. Biomaterials 33:13861395.

104. Schabitz WR, C Sommer, W Zoder, M Kiessling, M Schwaninger and S Schwab. (2000). Intravenous brainderived neurotrophic factor reduces infarct size and counterregulates Bax and Bcl-2 expression after temporary focal cerebral ischemia. Stroke 31:2212-2217.

105. Kiprianova I, J Sandkuhler, S Schwab, S Hoyer and M Spranger. (1999). Brain-derived neurotrophic factor improves long-term potentiation and cognitive functions after transient forebrain ischemia in the rat. Exp Neurol 159:511-519.

106. Failla MD, YP Conley and AK Wagner. (2015). Brainderived neurotrophic factor (BDNF) in traumatic brain injury-related mortality. Neurorehabil Neural Repair 30: 83-93.

107. Stanne TM, ND Aberg, S Nilsson, K Jood, C Blomstrand, $\mathrm{U}$ Andreasson, $\mathrm{K}$ Blennow, $\mathrm{H}$ Zetterberg, J Isgaard, J Svensson and C Jern. (2016). Low circulating acute brain-derived neurotrophic factor levels are associated with poor long-term functional outcome after ischemic stroke. Stroke 47:1943-1945.

108. Luo W, T Liu, S Li, H Wen, F Zhou, R Zafonte, X Luo, $\mathrm{M} \mathrm{Xu}, \mathrm{R}$ Black-Schaffer, et al. (2018). The serum BDNF level offers minimum predictive value for motor function recovery after stroke. Transl Stroke Res 10:342-351.

109. Xu B. (2013). BDNF (I)rising from exercise. Cell Metab 18:612-614. 
110. Pang J, J Peng, P Yang, L Kuai, L Chen, JH Zhang and Y Jiang. (2019). White matter injury in early brain injury after subarachnoid hemorrhage. Cell Transplant 28:26-35.

111. Wang H, YT Zhao, S Zhang, PM Dubielecka, J Du, N Yano, YE Chin, S Zhuang, G Qin and TC Zhao. (2017). Irisin plays a pivotal role to protect the heart against ischemia and reperfusion injury. J Cell Physiol 232:37753785 .

112. Gul-Kahraman K, M Yilmaz-Bozoglan and E Sahna. (2019). Physiological and pharmacological effects of melatonin on remote ischemic perconditioning after myocardial ischemia-reperfusion injury in rats: role of Cybb, Fas, NfкB, Irisin signaling pathway. J Pineal Res 67:e12589.

113. Shao L, D Meng, F Yang, H Song and D Tang. (2017). Irisin-mediated protective effect on LPS-induced acute lung injury via suppressing inflammation and apoptosis of alveolar epithelial cells. Biochem Biophy Res Commun 487:194-200.

114. Xu S-Z, S Liu, F Du, X Li, M Wang, R Duan, J Zhang, Y Wu and Q Zhang. (2017). Effects and underlying mechanisms of irisin on the proliferation and apoptosis of pancreatic $\beta$ cells. PloS One 12:e0175498.

115. Emami MR, E Alipoor and MJ Hosseinzadeh-Attar. (2017). Irisin - a potential contributor of insulin resistance in kidney failure. Eur J Inter Med 44:e22-e23.

116. Lu J, G Xiang, M Liu, W Mei, L Xiang and J Dong. (2015). Irisin protects against endothelial injury and ameliorates atherosclerosis in apolipoprotein E-Null diabetic mice. Atherosclerosis 243:438-448.

117. Galluzzi L, JM Bravo-San Pedro, K Blomgren and G Kroemer. (2016). Autophagy in acute brain injury. Nat Rev Neurosci 17:467-484.

118. Li X, J Peng, J Pang, Y Wu, X Huang, Y Li, J Zhou, L Gu, $\mathrm{X}$ Sun, et al. (2018). Apolipoprotein e-mimetic peptide COG1410 promotes autophagy by phosphorylating GSK$3 \beta$ in early brain injury following experimental subarachnoid hemorrhage. Front Neurosci 12:127.

119. Li R-L, S-S Wu, Y Wu, X-X Wang, H-Y Chen, J-J Xin, H Li, J Lan, K-Y Xue, et al. (2018). Irisin alleviates pressure overload-induced cardiac hypertrophy by inducing protective autophagy via mTOR-independent activation of the AMPK-ULK1 pathway. J Mol Cell Cardiol 121:242-255.

120. Li R, X Wang, S Wu, Y Wu, H Chen, J Xin, H Li, J Lan, $\mathrm{K}$ Xue, et al. (2019). Irisin ameliorates angiotensin IIinduced cardiomyocyte apoptosis through autophagy. J Cell Physiol 234:17578-17588.

121. Hashemi MS, K Ghaedi, A Salamian, K Karbalaie, M Emadi-Baygi, S Tanhaei, MH Nasr-Esfahani and H Baharvand. (2013). Fndc5 knockdown significantly decreased neural differentiation rate of mouse embryonic stem cells. Neuroscience 231:296-304.

122. Moon HS, F Dincer and CS Mantzoros. (2013). Pharmacological concentrations of irisin increase cell proliferation without influencing markers of neurite outgrowth and synaptogenesis in mouse H19-7 hippocampal cell lines. Metabolism 62:1131-1136.

123. Chen Z, A Iona, S Parish, Y Chen, Y Guo, F Bragg, L Yang, Z Bian, MV Holmes, et al. (2018). Adiposity and risk of ischaemic and haemorrhagic stroke in 0.5 million Chinese men and women: a prospective cohort study. Lancet Glob Health 6:e630-e640.
124. Makimattila S, K Malmberg-Ceder, AM Hakkinen, K Vuori, O Salonen, P Summanen, H Yki-Jarvinen, $\mathrm{M}$ Kaste, S Heikkinen, $\mathrm{N}$ Lundbom and RO Roine. (2004). Brain metabolic alterations in patients with type 1 diabetes-hyperglycemia-induced injury. J Cereb Blood Flow Metab 24:1393-1399.

125. Dale CE, G Fatemifar, TM Palmer, J White, D PrietoMerino, D Zabaneh, JEL Engmann, T Shah, A Wong, et al. (2017). Causal associations of adiposity and body fat distribution with coronary heart disease, stroke subtypes, and type 2 diabetes mellitus: a mendelian randomization analysis. Circulation 135:2373-2388.

126. Oddo M, JM Schmidt, E Carrera, N Badjatia, ES Connolly, M Presciutti, ND Ostapkovich, JM Levine, P Le Roux and SA Mayer. (2008). Impact of tight glycemic control on cerebral glucose metabolism after severe brain injury: a microdialysis study. Crit Care Med 36:32333238.

127. Langdon KD, J Clarke and D Corbett. (2011). Long-term exposure to high fat diet is bad for your brain: exacerbation of focal ischemic brain injury. Neuroscience 182: $82-87$.

128. Sarmah D, H Kaur, J Saraf, K Vats, K Pravalika, M Wanve, K Kalia, A Borah, A Kumar, et al. (2018). Mitochondrial dysfunction in stroke: implications of stem cell therapy. Transl Stroke Res [Epub ahead of print]; DOI: $10.1007 / \mathrm{s} 12975-018-0642-y$.

129. Khacho M, R Harris and RS Slack. (2019). Mitochondria as central regulators of neural stem cell fate and cognitive function. Nat Rev Neurosci 20:34-48.

130. Rajamani U, AR Gross, C Ocampo, AM Andres, RA Gottlieb and D Sareen. (2019). Retraction note: endocrine disruptors induce perturbations in endoplasmic reticulum and mitochondria of human pluripotent stem cell derivatives. Nat Commun 10:645.

131. Gu W, X Gaeta, A Sahakyan, AB Chan, CS Hong, R Kim, D Braas, K Plath, WE Lowry and HR Christofk. (2016). Glycolytic metabolism plays a functional role in regulating human pluripotent stem cell state. Cell Stem Cell 19: 476-490.

132. Knobloch M, SM Braun, L Zurkirchen, C von Schoultz, N Zamboni, MJ Arauzo-Bravo, WJ Kovacs, O Karalay, U Suter, et al. (2013). Metabolic control of adult neural stem cell activity by Fasn-dependent lipogenesis. Nature 493:226-230.

Address correspondence to: Yong Jiang, PhD

Department of Neurosurgery

The Affiliated Hospital of Southwest Medical University

No. 25 Taiping Street

Luzhou 646000

Sichuan

China

E-mail: jiangyong@swmu.edu.cn

Received for publication October 23, 2019

Accepted after revision January 7, 2020

Prepublished on Liebert Instant Online January 9, 2020 